Pymes manufactureras exportadoras en Colombia: un análisis de su actividad real

Fernando Mesa y Raúl Torres 
Lecturas de Economía, 90 (enero-junio 2019), pp. 127-157

Fernando Mesay Raúl Torres

Pymes manufactureras exportadoras en Colombia: un análisis de su actividad real

Resumen: Este artículo evalúa por qué algunas pequeñas y medianas empresas, o pymes, exportan y otras no, incluso cuando enfrentan situaciones macroeconómicas y de política comercial similares. Con este fin, se analizan estadísticamente las diferencias entre las pymes que son exportadoras y las que no lo son durante elperíodo 2000-2012. El análisis permite confirmar la modesta orientación de la estructura industrial colombiana hacia los mercados internacionales. Las pymes que exportaron crecieron más en su producción industrial que las no exportadoras; además, exbibieron los mayores tamaños en capital y número total de ocupados. Se verifica asi mismo la hipótesis sobre preselección de las pymes para exportar según niveles de productividad laboral. De igual manera, las empresas que registraron los mayores niveles de productividad presentaron las más altas probabilidades de exportar, aunque dicha probabilidad registró una tendencia decreciente en el periodo analizado. La probabilidad de exportar también se vio afectada positivamente por aquellas empresas que lo bicieron en el pasado.

Palabras clave: modelos de comercio con competencia imperfecta, economías de escala, estudios empiricos sobre comercio, empresas heterogéneas.

Clasificación JEL: F10, F12, F14.

Exporting industrial SMEs in Colombia: an analysis of their real activity

Abstract: This paper examines why some small and medium enterprises, or SMEs, export while others do not, even when they face similar macroeconomic and trade policy situations. With this aim, the difference between SMEs that are exporters and those that are not during the period 2000-2012 is analysed statistically. The analysis confirms the modest orientation of Colombia's industrial structure towards international markets. The SMEs that exported grew more in terms of industrial production than the non-exporting ones; in addition, they exhibited the greatest levels of capital and total number of employees. The hypothesis on pre-selection of SMEs to export according to labour productivity levels is also verified. Likewise, the companies that recorded the highest levels of productivity also presented the highest probabilities of exporting, although this probability registered a decreasing trend in the period analysed. The probability to export was also affected positively by those firms that did it in the past.

Keywords: models of trade with imperfect competition, economies of scale, empirical studies of trade, beterogenous firms.

JEL Classification: F10, F12, F14.

\section{Les PME manufacturières exportatrices en Colombie : une analyse de leur activité réelle}

Résumé: Cet article explique pourquoi certaines petites et moyennes entreprises(PME) sont exportatrices alors que d'autres ne le sont pas, même si elles se trouvent dans une situation macroéconomique et commerciale tout à fait similaire. À cette fin, nous analysons statistiquement les différences entre les PME exportatrices et les PME non-exportatrices au cours de la période 2000-2012. L'analyse confirme la faible orientation de la structure industrielle colombienne vers les marchés internationaux. Les PME exportatrices ont davantage développé leur production industrielle par rapport aux PME non-exportatrices. Les PME exportatrices ont un capital plus important et un nombre plus élevé des travailleurs. L’hypothèse de présélection des PME à exporter en fonction de la productivité du travail est également vérifiée. De même, les entreprises ayant enregistré les niveaux de productivité les plus élevés présentaient la probabilité d'exporter la plus élevée, bien que cette probabilité ait enregistré une tendance à la baisse sur la période analysée. La probabilité d'exporter a également été influencée positivement par les entreprises qui l'ont déjà fait.

Mots clés: modèle commercial avec concurrence imparfaite, économies d'échelle, études empiriques sur le commerce, entreprises hétérogènes.

Classification JEL: F10, F12, F14. 


\title{
Pymes manufactureras exportadoras en Colombia: un análisis de su actividad real
}

\author{
Fernando Mesa $\mathbb{D D}^{\mathrm{a}}$ y Raúl Torres (D) \\ -Introducción. -I. Evidencia empírica para las pymes. -II. Heterogeneidad de las pymes y la \\ probabilidad de exportar. -Conclusiones. -Referencias.
}

doi: 10.17533/udea.le.n90a05

Primera versión recibida el 27 de marzo de 2018; versión final aceptada el 23 de mayo de 2018

\section{Introducción}

Esta investigación analiza por qué algunas empresas pequeñas y medianas (pymes) exportan y otras no, incluso cuando todas enfrentan condiciones macroeconómicas y de política comercial similares. Para ello se examinan las variables reales en el ámbito microeconómico de las empresas, según la actividad económica de la Clasificación Internacional Industrial Uniforme (CIIU) y en consideración de si exportan o no.

En el análisis se verifica la hipótesis de que las pymes que serán exportadoras se diferencian de sus pares que no lo serán por registrar mayores productividades antes de hacerlo. Esta diferencia les permite a las primeras sufragar los costos de entrada a los mercados externos. Las variables de la productividad laboral, el tamaño de las empresas, el uso de capital físico, la proporción

a Fernando Mesa Parra: profesor, Departamento de Economía y Comercio Internacional, Universidad Jorge Tadeo Lozano. Dirección electrónica: fernandog.mesap@utadeo.edu.co. http:/ / orcid.org/0000-0002-6859-5165

b Raúl Torres Salamanca: profesor, Grupo de investigación de Empresas y Emprendimientos de Base Tecnológica, Escuela Colombiana de Ingeniería. Dirección electrónica: raul.torres@escuelaing.edu.co. http://orcid.org/0000-0003-2913-278X

Se agradece a los participantes de los seminarios internos del Departamento Nacional de Planeación de Colombia y a las universidades Jorge Tadeo Lozano, Universidad Pedagógica y Tecnológica de Tunja y la Escuela Colombiana de Ingeniería. También estamos en deuda con los comentarios y la ayuda que recibimos de Alejandro Mesa, Edwin Torres y Álvaro Zarta. 
Mesa y Torres: Pymes manufactureras exportadoras en Colombia: análisis de su actividad real

de trabajo no calificado con respecto al total de ocupados y su experiencia exportadora constituyen la base cuantitativa para estimar la probabilidad de que una pyme manufacturera de Colombia exporte.

El estudio se centra en las particularidades diferenciadoras de las pymes manufactureras exportadoras per se; esto es, no se presta atención a la dinámica ni a la distribución geográfica de las exportaciones de las pymes, ni tampoco a los efectos de la política comercial de fomento y de las variaciones de las cotizaciones cambiarias sobre las exportaciones en Colombia.

Las investigaciones basadas en microdatos para diferentes países e industrias focalizan su análisis en la producción y en la actividad exportadora de las empresas. Dichas investigaciones han encontrado que las empresas que exportan tienden a ser más productivas e intensivas en capital y, para el caso de los países menos desarrollados, tienden a emplear trabajo calificado más intensivamente y mejor remunerado (Bernard et al., 2003; Bernard \& Jensen, 2004; Bernard et al., 2007; Brooks, 2006; Melitz, 2008). En este estudio se considera el uso intensivo de trabajo no calificado, pero no las remuneraciones laborales, porque esto orientaría el escrito a examinar el mercado laboral.

Las variables fundamentales en la determinación de la heterogeneidad de las empresas pertenecientes a una misma actividad industrial son el tamaño y la productividad laboral. Esas diferencias son anteriores a la decisión de exportar, de tal manera que, como lo encontró Melitz (2008), las que se dirigen a los mercados internacionales gozan desde antes de ventajas productivas, lo que sugiere que existe un proceso de autoselección. En esta investigación se verifica empíricamente esta hipótesis de autoselección para las pymes manufactureras colombianas.

La mayoría de las investigaciones en Colombia relacionadas con el comportamiento de las exportaciones, tanto a nivel sectorial como de empresas, se orientan a explicar los determinantes económicos y su dinámica de crecimiento. Ha existido, sin embargo, menos interés en investigar específicamente las pymes, que por naturaleza tienen mayores desventajas para acceder a los mercados externos por sus rezagos tecnológicos y su menor capacidad de negociación y gestión. No obstante, estas podrían contar con ventajas sobre las empresas más grandes, por su mayor flexibilidad y velocidad operativa (Nassimbeni, 2001). 
A diferencia de las grandes empresas que cuentan con esquemas profesionales más apropiados para acceder a los mercados externos, las pymes dependen en alto grado de la capacidad gerencial de sus propietarios. Las limitaciones empresariales, la mayor aversión al riesgo y el limitado conocimiento de las oportunidades en los mercados extranjeros, reducen la habilidad de estas empresas para alcanzar las operaciones internacionales (Nassimbeni, 2001).

Recientemente se cuenta con amplia literatura teórica y empírica que explica el comercio internacional de acuerdo con las decisiones de empresas heterogéneas, en el sentido de la coexistencia en un mismo mercado de empresas grandes y pequeñas, así como de las diferencias de su eficiencia económica. Los trabajos pioneros en el estudio del comportamiento de las empresas heterogéneas en los mercados internacionales son los de Melitz (2003); Eaton, Kortum y Kramarz (2004); y Helpman, Melitz y Yeaple (2004).

Relacionados con el caso colombiano, desde una aproximación empírica, se han realizado estudios sobre los efectos de los costos y de las asimetrías en la entrada y a la salida de las empresas en los mercados externos (Roberts \& Tybout, 1997; Clerides, Lach \& Tybout, 1998). También se ha estudiado la desagregación de la dinámica de crecimiento y el destino de las exportaciones de las empresas, entre las permanentes y las que lo hacen transitoriamente (Eaton et al., 2007); así como las diferencias de las empresas que compiten con precios, o con la especialización de alguno de sus productos más competitivos, respecto a aquellas que lo hacen con calidad y variedades de sus productos en los mercados externos (Brooks, 2006). Bernard y Jensen (1999) encuentran, para los Estados Unidos, escasa evidencia de que las productividades aumentan sistemáticamente como resultado de tener acceso a los mercados externos, lo que coincide con lo encontrado por Clerides, Lach y Tybout (1998) para Colombia, México y Marruecos.

Los anteriores trabajos abordan el tema de las empresas exportadoras, pero no lo hacen bajo la problemática específica de las pymes exportadoras, sobre las cuales la investigación en Colombia es escasa. Esto se explica, en parte, por la insuficiente información estadística que existe en el país. Sobre esta temática se encuentran los estudios de Moori et al. (2005) y Milesi 
Mesa y Torres: Pymes manufactureras exportadoras en Colombia: análisis de su actividad real

y Aggio (2008). Las estadísticas utilizadas en los capítulos del Moori et al. (2005), dedicados al análisis de las pymes exportadoras en Colombia, se construyeron con base en encuestas realizadas antes de 2005. La publicación de las pymes exportadoras de Milesi y Aggio (2008) es a nivel latinoamericano, en donde se encuentra el caso colombiano y se desarrolla con la aplicación de índices. El estudio con encuestas de Uribe et al. (2006) enfatiza las ayudas financieras y las políticas de promoción exportadora en Colombia.

Miles et al. (2007) estudian los indicadores del éxito exportador de las pymes para Argentina, Chile y Colombia, a través de encuestas. Dichos indicadores incluyen la permanencia, diversificación y complejidad de productos, así como los mercados de exportación. El estudio encontró que la dimensión tecnológica fue un factor clave para determinar el éxito exportador de las pymes chilenas y argentinas, en tanto que el factor comercial lo fue para Colombia.

También cabe mencionar el trabajo de Meléndez (2013), que señala la baja inserción de las pymes de Colombia en los mercados externos y el limitado impacto de las políticas de promoción a las exportaciones; y, entre éstas, las de financiamiento. En Correa y Ortiz (2012), de otra parte, se analizan las pymes exportadoras con base en una encuesta y se estiman sus probabilidades de exportar de acuerdo con el desempeño financiero.

Para este estudio se construye un "panel" de pymes, con el fin de contrastar las características de las empresas exportadoras frente a las que no lo son, de acuerdo con el tamaño, la acumulación de capital, la productividad por trabajador y la composición de la fuerza laboral en términos del personal ocupado en producción en relación con el total de los ocupados. La información estadística para el período 2000-2012 se tomó de la Encuesta Anual Manufacturera (EAM) del Departamento Nacional de Estadística (DANE). Cabe destacar que infortunadamente la información de la EAM tiene reserva estadística y, por lo tanto, el DANE no autoriza cruzar la información estadística que se encuentra en la EAM con la de comercio exterior, que permitiría establecer los productos y los destinos de las exportaciones de las pymes exportadoras. 
Para identificar las diferencias entre las empresas exportadoras y las que no lo son, en el período 2000-2012, se sigue la estrategia econométrica utilizada en Bernard y Jensen (1999). La idea básica es diferenciar las pymes entre las que exportan y las que no lo hacen, de acuerdo con variables reales, para establecer el papel que juegan las características de heterogeneidad de las que exportan.

La metodología que se sigue para verificar la hipótesis de la existencia de la autoselección de las pymes exportadoras en Colombia, antes de serlo, es la siguiente. Primero se seleccionan las pymes que no exportaban al principio de dos subperíodos, 2000-2006 y 2007-2012; luego se verifica econométricamente para cada subperiodo si realmente existió mayor productividad laboral de las empresas que exportarían en el subperíodo, en relación con las que no lo harían.

Por la existencia de la heterogeneidad de las pymes, donde la productividad laboral juega un papel fundamental en la explicación de la orientación exportadora de las empresas, explica el interés de estimar la probabilidad que tiene una empresa en particular para exportar. Estas estimaciones se realizan con los modelos de variables dicotómicas, como es el probit.

El estudio confirma la modesta orientación de la estructura industrial colombiana hacia los mercados internacionales, en este caso de las pymes, como también de la existencia de una preselección de las que exportarán, según sus niveles de productividad laboral alcanzados previamente en el mercado nacional. Con la característica diferenciadora de la productividad por ocupado de las pymes exportadoras, se muestra que éstas crecieron más en términos de su producción industrial, fueron las que presentaron los mayores tamaños en términos de capital y número total de ocupados y registraron las menores relaciones de trabajo no calificado a calificado. No es sorprendente también encontrar que el éxito pasado de una pyme en su actividad exportadora sea el mejor indicador para que en el futuro lo siga siendo.

Adicional a esta introducción se tienen tres secciones adicionales. En la siguiente sección se relacionan las estadísticas descriptivas de las pymes según la EAM. En la tercera sección se describen las aproximaciones econométricas usadas y se muestran los resultados de las estimaciones, relacionados 
Mesa y Torres: Pymes manufactureras exportadoras en Colombia: análisis de su actividad real

con la heterogeneidad de las pymes exportadoras en Colombia; las mayores productividades por ocupado que registran las que exportarían al final de un subperíodo; y la probabilidad que existe de que una pyme en Colombia exporte según su productividad laboral y su pasada experiencia exportadora. La última sección presenta las conclusiones.

\section{Evidencia empírica para las pymes}

Para el período 2000-20012 se seleccionaron las empresas que contaban hasta con 50 ocupados de la EAM del DANE. Cabe destacar que las estadísticas de la encuesta del DANE son a nivel de plantas, pero para el propósito del estudio de las pymes se agregan a nivel de empresas.

El grupo de empresas, que tiene hasta 50 ocupados, las llamamos pymes; no obstante, que, en sentido estricto, las empresas medianas se clasifican usualmente hasta los 150 ocupados. Este criterio no se sigue aquí porque implicaría que las empresas seleccionadas en el estudio estarían cercanas al total de las empresas encuestadas por el DANE. Además, para el caso colombiano, las empresas con más de 50 empleados podrían considerarse como relativamente grandes.

Los propósitos de esta sección son dos: el primero es describir la evolución y la participación del número total de las pymes exportadoras durante el período señalado; el segundo, es presentar la participación que han tenido las exportaciones de las pymes, en relación con sus ventas totales.

Para mostrar la importancia de las pymes en la EAM del DANE, la Tabla 1 relaciona las estadísticas del total de estas empresas que encuesta el DANE para el período 2000-2012. El total de las empresas encuestadas en 2012 fue de 9.243 , con un crecimiento promedio anual de 1,6\% en el período. La participación promedio de las pymes en el total de las empresas encuestadas por el DANE fue de $71,0 \%$ y su crecimiento promedio anual fue igual al del total de empresas, de 1,0\% (Tabla 1).

El Gráfico 1 muestra la participación de las empresas exportadoras sobre el total de las encuestadas, que fue, en promedio, de 23,4\%; mientras que el porcentaje de las pymes exportadoras fue significativamente menor, de 
13,2\%. Así mismo, este gráfico muestra una caída en la participación de las empresas exportadoras durante el período, tanto para el total de ellas como para las pymes.

Tabla 1. Total de empresas y de pymes manufactureras y exportadoras en Colombia

\begin{tabular}{cccc}
\hline Año & $\begin{array}{c}\text { Total empresas } \\
\text { encuestadas (1) }\end{array}$ & Pymes (2) & $\begin{array}{c}\text { Participación pymes en total } \\
\text { empresas }(\%)(3)=(2) /(1)\end{array}$ \\
\hline 2000 & 7643 & 5601 & 73,3 \\
2001 & 7301 & 5261 & 72,1 \\
2002 & 7191 & 5106 & 71,0 \\
2003 & 7293 & 5147 & 70,6 \\
2004 & 7273 & 5054 & 69,5 \\
2005 & 7533 & 5234 & 69,5 \\
2006 & 7243 & 4922 & 68,0 \\
2007 & 7110 & 4733 & 66,6 \\
2008 & 7828 & 5355 & 68,4 \\
2009 & 9070 & 6634 & 73,1 \\
2010 & 9823 & 7306 & 74,4 \\
2011 & 9622 & 7084 & 73,6 \\
2012 & 9243 & 6701 & 72,5 \\
\hline
\end{tabular}

Nota: se definen las pymes como las empresas que tienen hasta 50 ocupados.

Fuente: estimaciones con base en la EAM del DANE.

La composición de las pymes, según su tamaño medido por el número de ocupados, se relaciona en el Gráfico 2. Las más pequeñas, con menos de 20 ocupados, representaron en promedio el 65,8\% del total de las pymes. Las de mayor tamaño, más de 40 ocupados, representaron el 7,5\%. La participación de las pymes es menor a medida que aumenta su tamaño.

Las pymes exportadoras más pequeñas, hasta 20 ocupados, fueron en promedio el 8,1\% de las empresas de ese tamaño, en tanto que las pymes exportadoras de mayor tamaño, con más de 40 ocupados, fueron en promedio el 29,3\% (Gráfico 3). Para las pymes esos porcentajes promedios caen a partir del 2004. Se destaca que la participación de las pymes exportadoras es menor entre más pequeñas son. De otro lado, la caída de la participación de las pymes 
Mesa y Torres: Pymes manufactureras exportadoras en Colombia: análisis de su actividad real

exportadoras en el 2004 podría explicarse por la reactivación de la demanda interna y por la inestabilidad de sus mercados en los países fronterizos.

Gráfico 1. Participación de las empresas exportadoras (\%)



Fuente: estimaciones con base en la EAM del DANE.

Gráfico 2. Composición de las pymes según su tamaño

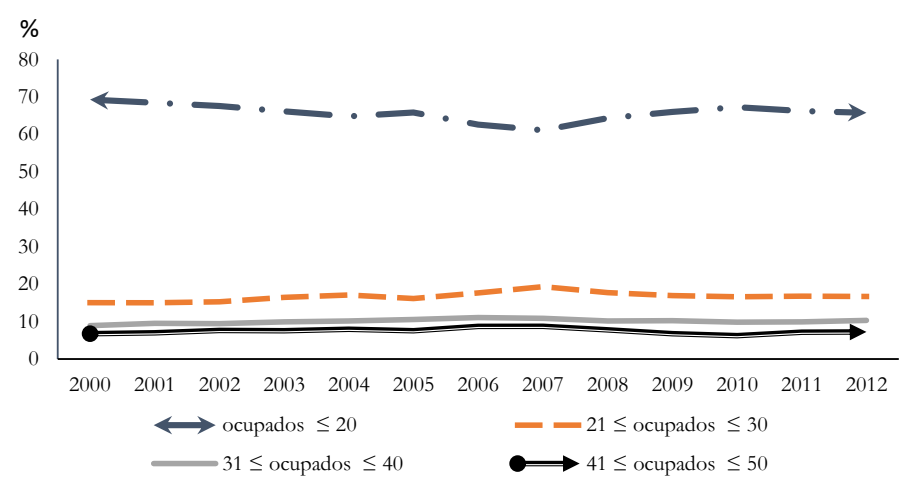

Fuente: estimaciones con base en la EAM del DANE.

Para el período 2000-2012, la participación promedio de las exportaciones en las ventas totales de las pymes que exportan fue 13,2\%. Sin embargo, para aquellas que tenían entre 21 y 30 ocupados el promedio fue superior, $22,4 \%$ (Gráfico 4). De otra parte, las pymes de mayor tamaño, entre 41 y 50 ocupados, que debieron haber presentado las mayores participaciones de 
exportaciones, sorprendentemente tuvieron un porcentaje más bajo $(11,2 \%)$ en comparación con las de menor tamaño, como fueron las de entre 21 y 30 ocupados. Cabe destacar que la participación del valor de las exportaciones en las ventas de las pymes entre 21 y 30 ocupados también fue la más inestable durante el período, según el coeficiente de variación (Gráfico 4).

Gráfico 3. Participación de las pymes exportadoras en el total de las pymes según su tamaño

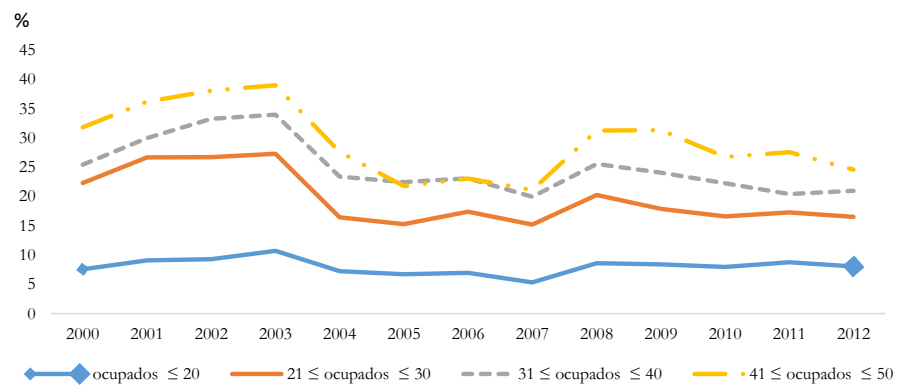

Fuente: estimaciones con base en la EAM del DANE.

Gráfico 4. Participación de las exportaciones en las ventas totales de las pymes exportadoras

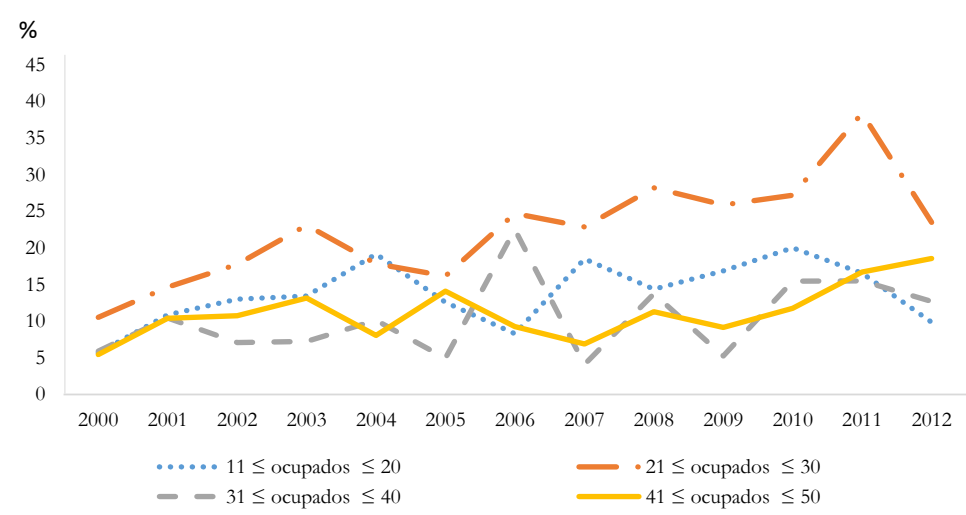

Fuente: estimaciones con base en la EAM del DANE.

El número de las pymes exportadoras a nivel de las actividades industriales se relaciona en la Tabla 2, para los años 2000, 2005, 2010 y 2012. Las 
Mesa y Torres: Pymes manufactureras exportadoras en Colombia: análisis de su actividad real

ramas industriales que mostraron los mayores porcentajes de empresas exportadoras en 2012 fueron: cuero y calzado (22,8 \%), papel y sus productos $(19,4 \%)$, metalúrgicas básicas $(18,4 \%)$, fabricación de maquinaria y equipo $(18,2 \%)$, maquinaria de oficina y eléctricos $(17,6 \%)$, aparatos de radio y televisión $(25,9 \%)$, y equipo de transporte $(19,5 \%)$, esta última con tendencia creciente. La participación de las pymes que exportaban textiles cayó en los años que se reportan. Las anteriores ramas industriales representaron el 30\% del total de las pymes exportadoras.

Las ramas que registraron las menores participaciones de empresas en el mercado externo en 2012 fueron: alimentos, bebidas y tabaco $(5,1 \%)$; madera y sus productos $(5,6 \%)$; productos minerales no metálicos $(6,5 \%)$; y productos de metal, excepto maquinaria $(7,7 \%)$. Estas actividades representaron el $15,9 \%$ del total de las pymes exportadoras (véase Tabla 2).

Por su parte, las pymes en las industrias de alimentos, bebidas y tabaco, con una participación relativamente baja de empresas exportadoras en 2012 $(5,2 \%)$, presentaron mayor participación del valor exportado en las ventas totales en ese año, que fue 10,6\%. Las de la industria metalúrgica básica, con $18,4 \%$ de empresas exportadoras en el 2012 , alcanzaron a exportar $82,8 \%$ de sus ventas totales. Al contrario, las pymes de la actividad de vehículos automotores, que relativamente presentaron alto porcentaje de empresas exportadoras $(14,8 \%)$, tuvieron una participación del valor de las exportaciones dentro de las ventas totales significativamente baja (3,5\%) en el 2012 (véase Tabla 3).

En el 2010 se registró un relativo alto porcentaje de exportaciones con respecto a las ventas totales de las empresas (18,9\%), comparado con los otros tres años. Ese mayor porcentaje se explica por la alta participación de las exportaciones en: alimentos y bebidas, papel y sus productos, productos metalúrgicos básicos, aparatos de radio y televisión, y equipos de transporte.

Los resultados anteriores confirman la distribución bimodal que señala Brooks (2006) de las empresas exportadoras en Colombia, en el sentido que un alto porcentaje de las empresas son de baja intensidad exportadora, en tanto que un pequeño porcentaje de empresas son de mayor intensidad exportadora. Entre las últimas lo fueron en 2012 las actividades de alimentos y bebidas, papel y sus productos, y metalúrgica básica. 


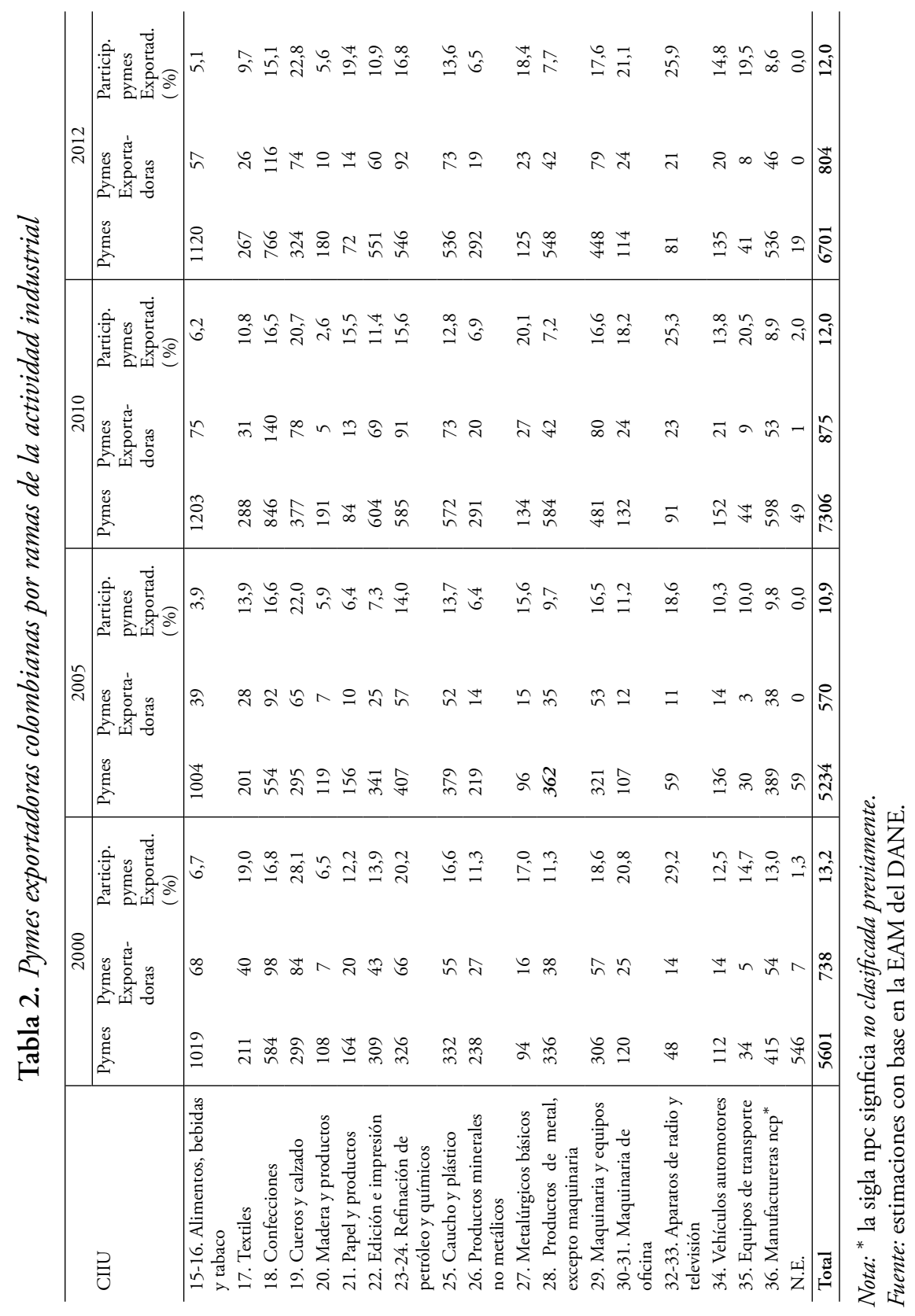


Mesa y Torres: Pymes manufactureras exportadoras en Colombia: análisis de su actividad real

Tabla 3. Participación del valor exportado en el total de las ventas de las pymes exportadoras (\%)

\begin{tabular}{lcccc}
\hline CIIU & 2000 & 2005 & 2010 & 2012 \\
\hline 15-16. Alimentos, bebidas y tabaco & 7,8 & 7,4 & 13,8 & 10,6 \\
17. Textiles & 1,5 & 11,4 & 6,2 & 8,2 \\
18. Confecciones & 4,8 & 11,5 & 9,7 & 8,4 \\
19. Cueros y calzado & 5,8 & 11,2 & 8,7 & 9,5 \\
20. Madera y productos & 0,5 & 2,8 & 0,2 & 1,1 \\
21. Papel y productos & 6,5 & 1,8 & 19,2 & 18,3 \\
22. Edición e impresión & 8,1 & 13,3 & 3,5 & 3,7 \\
23-24. Refinación de petróleo y químicos & 5,5 & 16,1 & 6,4 & 1,2 \\
25. Caucho y plástico & 2,1 & 2,8 & 2,2 & 3,1 \\
26. Productos minerales no metálicos & 0,9 & 1,3 & 0,8 & 1,5 \\
27. Metalúrgicos básicos & 20,5 & 77,5 & 94,0 & 82,8 \\
28. Productos de metal, excepto maquinaria & 4,9 & 11,5 & 6,0 & 6,4 \\
29. Maquinaria & 6,2 & 8,6 & 4,6 & 5,6 \\
30-31. Maquinaria de oficina y eléctricos & 4,3 & 8,8 & 10,4 & 4,0 \\
32-33. Aparatos de radio y televisión & 5,0 & 6,4 & 19,7 & 7,5 \\
34. Vehículos automotores & 5,3 & 4,6 & 3,6 & 3,5 \\
35. Equipos de transporte & 6,3 & 1,5 & 11,8 & 5,9 \\
36. Manufactureras ncp* & 3,6 & 4,9 & 4,2 & 4,6 \\
N.E. & 1,8 & 0,0 & 0,4 & 0,0 \\
Total & 6,6 & 10,5 & 18,9 & 9,1 \\
\hline
\end{tabular}

Nota: ${ }^{*}$ la sigla npc signficia no clasificada previamente.

Fuente: estimaciones con base en la EAM del DANE.

Las Tablas 4 y 5 relacionan la permanencia en el mercado externo y la participación de las exportaciones en las ventas totales de las pymes según su cohorte de entrada. Algunas de las que permanecen, y que se registran en las tablas en referencia, podrían dejar de ser pymes debido a su mayor tamaño. En general, el grado de persistencia de las pymes exportadoras es bajo. Se aclara que no se incorporan en las tablas la información para los años 2011 y 2012, porque el interés es mostrar la permanencia de las pymes como exportadoras. 


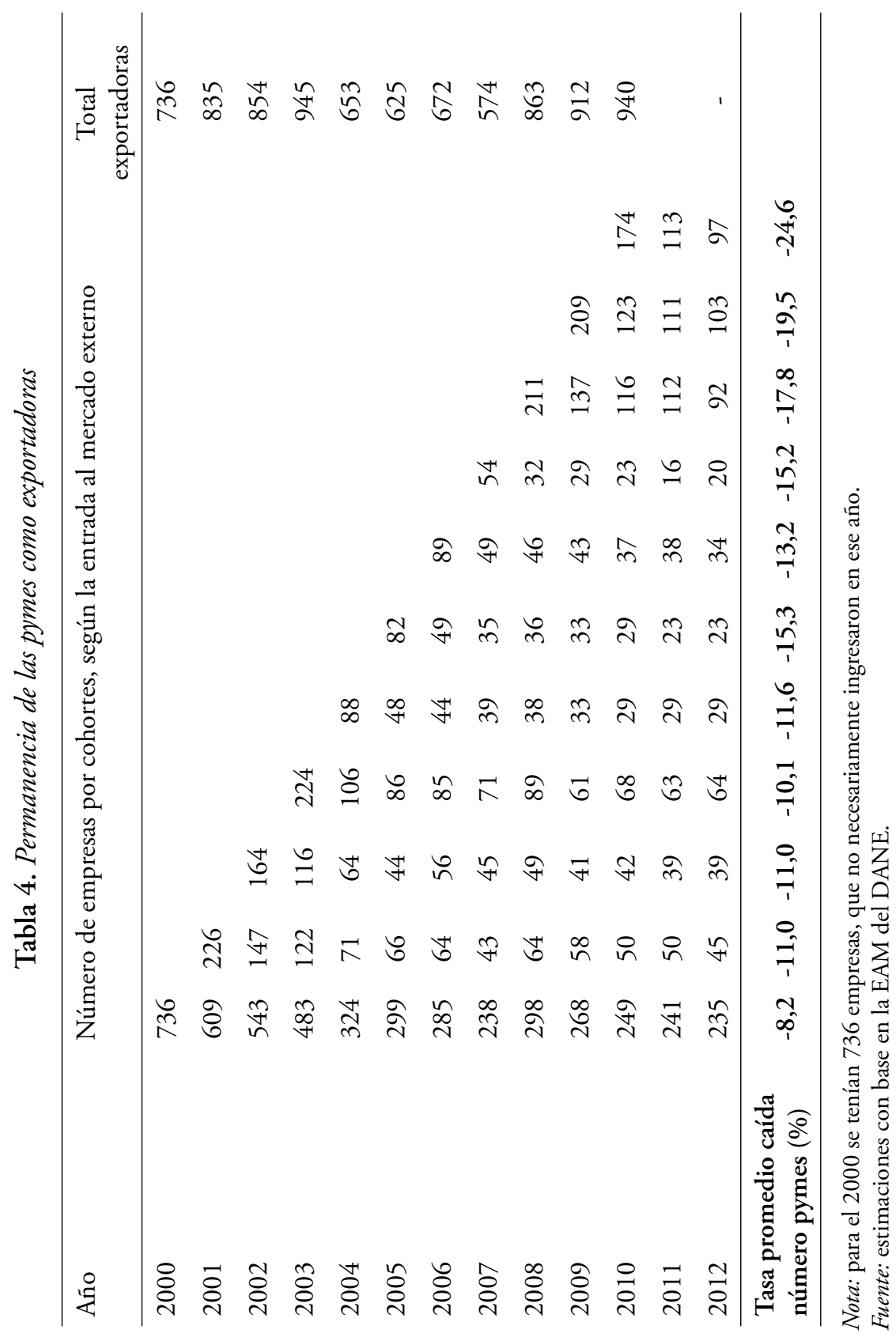


Mesa y Torres: Pymes manufactureras exportadoras en Colombia: análisis de su actividad real

Tabla 5. Exportaciones sobre el total de ventas de las pymes exportadoras por cohortes de entrada

\begin{tabular}{ccccccccccccc}
\hline Año & \multicolumn{1}{c}{ Porcentaje de las exportaciones por cohorte de entrada al mercado externo (\%) } \\
\hline 2000 & 6,6 & & & & & & & & & \\
2001 & 12,6 & 12,3 & & & & & & & & \\
2002 & 10,5 & 10,1 & 23,7 & & & & & & & \\
2003 & 12,0 & 12,2 & 32,6 & 10,5 & & & & & & \\
2004 & 17,3 & 16,2 & 22,1 & 7,9 & 12,7 & & & & & \\
2005 & 11,4 & 12,2 & 20,6 & 2,9 & 9,7 & 4,4 & & & & \\
2006 & 20,1 & 19,3 & 20,4 & 3,4 & 10,0 & 3,3 & 3,4 & & & \\
2007 & 23,5 & 22,0 & 11,8 & 2,3 & 9,7 & 9,1 & 7,8 & 2,1 & & & \\
2008 & 17,1 & 18,5 & 7,9 & 1,3 & 18,7 & 2,6 & 4,8 & 25,9 & 15,1 & & \\
2009 & 20,2 & 21,2 & 5,2 & 12,6 & 9,1 & 4,2 & 8,1 & 26,0 & 25,0 & 23,6 & \\
2010 & 22,3 & 23,2 & 9,0 & 3,4 & 12,0 & 2,4 & 1,9 & 25,3 & 13,8 & 30,8 & 12,0 \\
2011 & 25,1 & 12,4 & 20,3 & 6,4 & 9,8 & 1,1 & 6,3 & 2,4 & 21,6 & 33,9 & 8,7 \\
2012 & 16,6 & 9,3 & 7,3 & 7,3 & 8,8 & 4,1 & 1,2 & 0,6 & 25,0 & 38,9 & 24,1 \\
\hline
\end{tabular}

Fuente: estimaciones con base en la EAM del DANE.

La disminución del número de empresas exportadoras por cohortes, de acuerdo con su año de entrada al mercado externo, es variable y no existe un patrón determinado (Tabla 4). La mayor permanencia relativa se dio con la cohorte de las 224 empresas que entraron en 2003, de las cuales solo exportaban 63 en el 2012, con una tasa promedia anual de salida del mercado externo de 10,1\%. De las 736 empresas exportadoras que se registraron en la cohorte del 2000, solo 235 lo seguían haciendo en 2012, con una tasa promedia anual de salida al mercado externo de $8,2 \%$. Se esperaría que entre mayor sea el número de años que las empresas exporten, mayor es la probabilidad de su supervivencia en el mercado externo, por lo que es previsible que en los primeros años salga el mayor número de empresas.

Se destaca el aumento de 289 empresas exportadoras en el 2008, al computar todas las cohortes en la última columna de la Tabla 4. Ese número se explica por las 211 nuevas pymes que iniciaron sus exportaciones en ese año, más 
70 que entraron nuevamente a exportar de la cohorte del 2000 y otras 8 en diferentes cohortes. Es importante precisar que el mayor número de empresas exportadoras en la cohorte del año 2000 se explica porque se contabilizan empresas que habían iniciado sus exportaciones antes del 2000.

La Tabla 5 evidencia la baja participación de las exportaciones en relación con las ventas totales de las pymes exportadoras. Cabe señalar, sin embargo, que no existe un claro aumento de la intensidad exportadora de las empresas con mayor permanencia en los mercados internacionales. Las que entraron en el mercado externo en el 2002 y en años posteriores redujeron la participación de las exportaciones.

Esta evidencia empírica permite deducir que la mayor contribución a las exportaciones proviene de las empresas ya establecidas en los mercados externos y marginalmente tanto por las que entran recientemente como por las que salen con el tiempo. Son notorias las altas y volátiles participaciones de las exportaciones de las últimas cohortes. Estos resultados son consistentes con los resultados del trabajo de Eaton et al. (2007).

\section{Heterogeneidad de las pymes y su probabilidad de exportar}

Esta sección describe primero las variables de las pymes que se usan en los modelos que se estiman. Luego se analizan econométricamente los patrones que diferencian a las pymes que exportaron de las que no lo hicieron. A continuación, se verifica la hipótesis de que las pymes con mayores niveles de productividad son las que exportan en el futuro; dicha verificación se realiza para los subperíodos 2000-2006 y 2007-2012. Al final de la sección se estiman las probabilidades de que una pyme exporte, considerando si exportó en el pasado, sus niveles de productividad laboral y otras variables reales de control.

\section{A. Descripción de variables}

Con la información estadística por empresa se construye el siguiente conjunto de variables estructurales continuas, que son: el numero de ocupados, la producción real industrial, el nivel de capital y la productividad por ocupado. Se calcula la 
Mesa y Torres: Pymes manufactureras exportadoras en Colombia: análisis de su actividad real

relación entre los ocupados no calificados y el total de ocupados, donde los no calificados son los ocupados directamente en la producción. Esta última relación mide indirectamente la incorporación de nuevas tecnologías en los sistemas de producción de las empresas, de tal manera que entre mayor es el coeficiente menor es el uso de nuevas tecnologías que involucran las empresas.

La productividad por ocupado se calcula como la relación entre el valor agregado a precios constantes, o entre la producción bruta industrial igualmente a precios constantes, y el total de los ocupados. Para expresar el valor agregado y el de la producción industrial a precios constantes se utilizan los índices de precios al productor del DANE.

Algunas dimensiones que diferenciaron a las pymes que exportaron en relación con las que no lo hicieron para el período 2000-2012 se relacionan en la Tabla 6. En efecto, las pymes exportadoras ocuparon en promedio 25,3 personas y las no exportadoras 16,9. La relación entre ocupados en producción y el total de ocupados fue menor para las exportadoras $(0,59)$ frente a las no exportadoras $(0,62)$. Lo anterior fue consistente con el mayor uso intensivo de capital de las pymes exportadoras, el cual se toma con el logaritmo de los niveles de capital.

Basta señalar que las diferencias de medias entre las variables de las empresas que exportaron y las que no lo hicieron son estadísticamente significativas al $1 \%$ (Tabla 6). Además, las pymes exportadoras representaron el 13,1\% del total de las empresas de este tipo, y el valor obtenido por exportación, en relación con el total de sus ventas, fue $29 \%$.

Se destaca el mayor nivel del logaritmo de la productividad laboral de las pymes que exportaron con respecto a su contraparte. El Gráfico 5 muestra la tendencia creciente promedio de dicho logaritmo (en términos del valor agregado) de las pymes exportadoras y no exportadoras, siendo el logaritmo de las primeras el que registra niveles más altos.

La mayor productividad de las pymes exportadoras implica que estas empresas presentaron menores costos unitarios de producción. Si las barreras comerciales de entrada a los mercados externos fueran iguales para todas las pymes, esa diferencia en los costos unitarios sería el factor que determinaría que una empresa exporte y a cuál mercado de destino. 


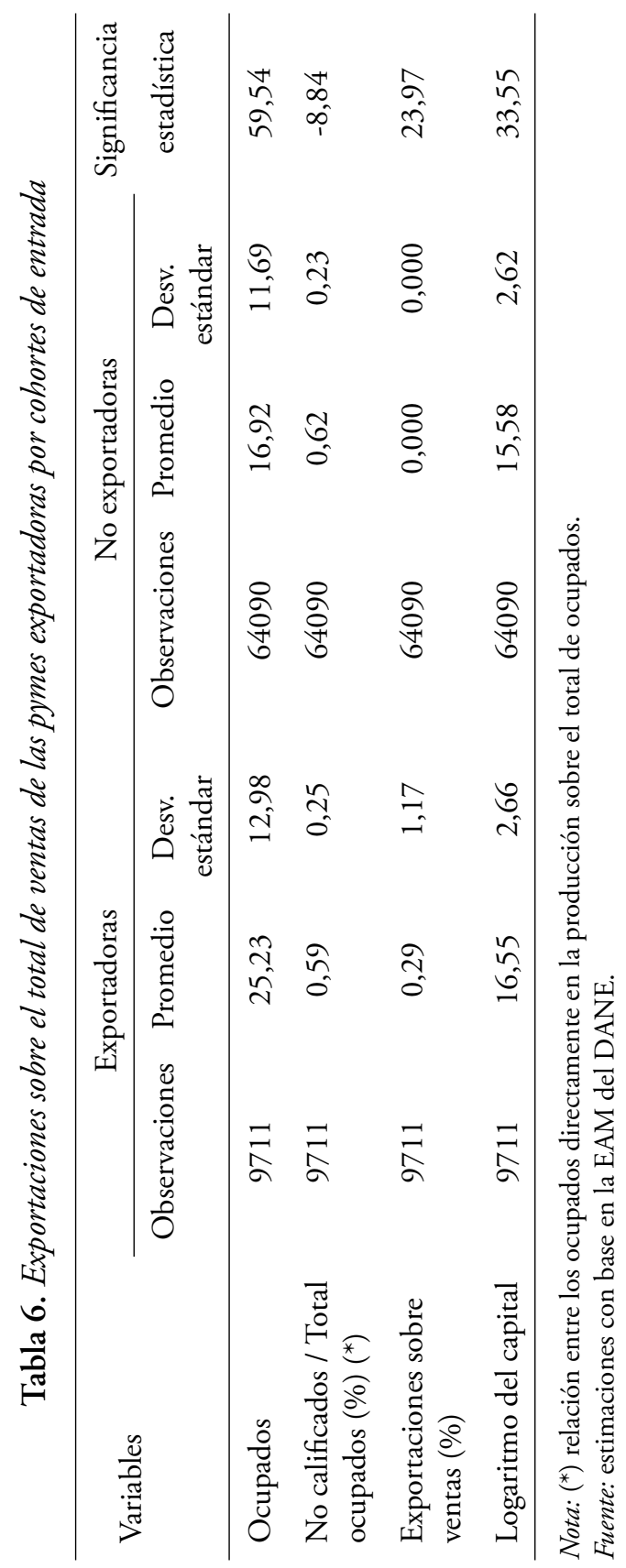


Mesa y Torres: Pymes manufactureras exportadoras en Colombia: análisis de su actividad real

Gráfico 5. Logaritmo de la productividad laboral en valor agregado real, 2000-2012

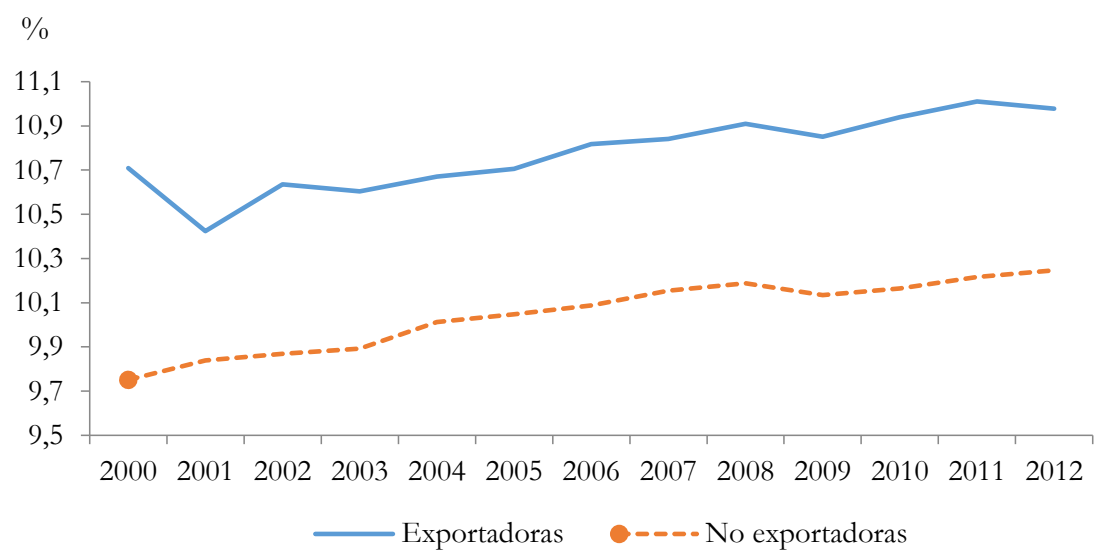

Fuente: estimaciones con base en la EAM del DANE.

\section{B. Prima de desempeño de las pymes exportadoras}

Para examinar si existen diferencias económicas sistemáticas entre las pymes que exportaron y las que no lo hicieron durante el período 2000-2012, se sigue la aproximación metodológica de Bernard y Jensen (1999), la cual también fue utilizada en los trabajos de Bernard et al. (2007) y Lawless (2009). De esta manera se procede a estimar ocho paneles con la expresión

$$
\ln X_{i t}=\beta_{0}+\beta_{1} * \text { Expo }_{i t}+\beta_{2} * X+\beta_{3} * C I I U_{i}+e_{i},
$$

donde el subíndice $i$ identifica la pyme y $t$ el tiempo; X es el vector de las características económicas de las empresas; Expo es una dummy igual a 1 si exporta e igual a 0 si no lo hace; y CIIU son 22 dummies de actividad económica. El $e_{i}$ es el error de estimación. El parámetro $\beta_{1}$ muestra las diferencias porcentuales entre las pymes que exportaron y las que no lo hicieron dentro de una misma actividad industrial. El vector $\mathrm{X}$ de variables que controlan las características de las empresas son: el tamaño de las empresas, medido por los logaritmos del capital o de los ocupados, y la relación entre los ocupados en producción con respecto al total de ocupados. Los resultados de las estimaciones se presentan en la Tabla 7. 





Mesa y Torres: Pymes manufactureras exportadoras en Colombia: análisis de su actividad real

Se indica que los parámetros estimados son significativos al $1 \%$ y los signos permiten explicar las diferencias sistemáticas que presentaron las pymes exportadoras en el período (Tabla 7). Contrario a los resultados de la Tabla 6, en este ejercicio se muestran las diferencias de las empresas que exportaron, después de depurar esas diferencias con las variables de control.

La regresión (1), controlada por el tamaño de los ocupados, indica que las pymes exportadoras produjeron $19,2 \%$ más que las no exportadoras; mientras que la regresión (2), controlada por el logaritmo del capital, indica que las exportadoras produjeron $35,0 \%$ más. Los parámetros relacionados con los logaritmos de las variables de control se leen como elasticidades.

La regresión (3) indica que las pymes exportadoras ocuparon en promedio $14,9 \%$ más trabajadores que las no exportadoras. El parámetro positivo del logaritmo del capital indica que su aumento en uno, los ocupados los harían en $0,18 \%$.

Por otro lado, la regresión (4) confirma el resultado de la Tabla 6 con respecto al mayor uso de capital por parte de las pymes exportadoras. Según esta regresión, las exportadoras registraron $11,3 \%$ más capital que las no exportadoras. También es consistente con el signo negativo del parámetro que relaciona la proporción de los ocupados en producción con el logaritmo del capital. De todas maneras, los mayores niveles de capital y el uso de las economías de escala de las pymes exportadoras les permitió crecer más (cerca de 5,5 puntos porcentuales más en términos de producción bruta industrial en promedio durante el período) y mostrar mayores niveles de productividad que las pymes no exportadoras (Roberts \& Tybout, 1997).

Las cuatro últimas regresiones - de la (5) a la (8)- confirman la mayor productividad laboral de las pymes exportadoras. Si la productividad laboral se mide en términos de valor agregado, esas diferencias se encuentran entre un 21,3 y un $25,0 \%$, dependiendo de las variables de control incluidas -regresiones (5) y (6)-. En cambio, si la productividad laboral se mide en términos de la producción industrial bruta, esos valores se encuentran entre 16,6 y 19,2\%, dependiendo también de las variables de control incluidas en la estimación. Vale la pena resaltar el parámetro positivo del logaritmo del uso del capital y el negativo de la proporción entre los trabajadores en producción y el total. 


\section{Autoselección de las pymes exportadoras según sus niveles de productividad}

Los estudios de las industrias de los países confirman que las altas productividades preceden a la entrada de los mercados externos (Bernard et al., 2007). Esos resultados sugieren que la presencia de los costos de entrada en los mercados de exportación solo permite que las empresas más productivas puedan sufragarlos, como lo enfatizan Roberts y Tybout (1997). Para verificar empíricamente la hipótesis de que las pymes exportadoras presentaban mayores niveles de productividad laboral en relación con las no exportadoras, se sigue la metodología de Bernard y Jensen (1999). Esta consiste en seleccionar las pymes que no exportaron en el primer año de cada uno de los subperiodos 2000-2006 y 2007-2012 y luego comparar los niveles de las productividades laborales entre las pymes que exportarían en el futuro con los niveles de las que no lo harían, según la expresión

$$
\log \varphi_{i 0}=\beta_{0}+\beta_{1} E X P O R T A_{i}+\beta_{2} X_{i 0}+\beta_{3} C I I U_{i}+\varepsilon_{i},
$$

donde $\varphi_{i 0}$ es la productividad laboral de la pyme i al principio de cada subperíodo; EXPORT $A_{i T}$ es la dummy con valor de 1 si la pyme exportaría en el período y 0 si no lo haría; $X_{i 0}$ es el logaritmo del número de ocupados o la proporción de ocupados en producción con respecto al total a principio del período; y CIIU son dummies de la actividad económica de la correspondiente pyme. El parámetro $\beta_{1}$ mide la mayor productividad laboral, en este caso con respecto al valor agregado o a la producción bruta industrial, de las futuras pymes exportadoras.

Las regresiones (1) a (4) (Tabla 8) muestran sin ambigüedad que las productividades laborales fueron mayores para las pymes que exportarían en cada subperíodo, y entre éstas, las productividades de las pymes que exportarían en el segundo período (2007-2012) fueron mayores que las del primero (20002006). En las estimaciones se tomaron como variables de control el tamaño de las empresas, de acuerdo con el logaritmo del total de los ocupados, y la relación entre ocupados en producción y su total. El logaritmo del total de ocupados no fue significativo o, en caso de serlo, no se obtuvo un signo único. 
Mesa y Torres: Pymes manufactureras exportadoras en Colombia: análisis de su actividad real

Tabla 8. Verificación de la hipótesis: las pymes exportadoras tienen mayores productividades laborales que las no exportadoras

\begin{tabular}{lcccc}
\hline & $(1)$ & $(2)$ & $(3)$ & $(4)$ \\
Variables & $\begin{array}{l}\text { Log. productividad } \\
\text { en valor agregado }\end{array}$ & $\begin{array}{c}\text { Log. productividad en } \\
\text { producción industrial }\end{array}$ & $\begin{array}{l}\text { Log. productividad } \\
\text { en valor agregado }\end{array}$ & $\begin{array}{c}\text { Log. productividad en } \\
\text { producción industrial }\end{array}$ \\
\cline { 2 - 5 } & \multicolumn{2}{c}{ Período $2000-2006$} & Período 2007 - 2012 \\
\hline Exportaciones & $0,362^{* * *}$ & $0,379^{* * *}$ & $0,444^{* * *}$ & $0,380^{* * *}$ \\
Log. total ocupados & $(0,083)$ & $(0,079)$ & $(0,068)$ & $(0,058)$ \\
& $0,159^{* * *}$ & $-0,016$ & 0,038 & $-0,043^{* *}$ \\
No calificados/Total ocupados & $(0,025)$ & $(0,023)$ & $(0,025)$ & $(0,021)$ \\
Dummies de actividad económica & $-0,367^{* * *}$ & $-0,320^{* * *}$ & $-0,446^{* * *}$ & $-0,428^{* * *}$ \\
Constante & 23 Dummies & 23 Dummies & 23 Dummies & 23 Dummies \\
Observaciones & $9,201^{* * *}$ & $10,757^{* * *}$ & $9,701^{* * *}$ & $11,076^{* * *}$ \\
R-cuadrado & $(0,077)$ & $(0,073)$ & $(0,082)$ & $(0,070)$ \\
\hline
\end{tabular}

Nota: errores estándar entre paréntesis. ${ }^{* * *} \mathrm{p}<0,01,{ }^{* *} \mathrm{p}<0,05,{ }^{*} \mathrm{p}<0,1$.

Fuente: estimaciones con base en la EAM del DANE.

Como lo señalan Clerides, Lach y Tybout (1998), las empresas que entran a los mercados externos generalmente presentan mejor desempeño económico que las que no lo hacen. La productividad laboral y el uso más intensivo de trabajo calificado se incrementan antes de iniciar las exportaciones y, como también lo anotan los autores, esas mejoras no se originan ni se desarrollan cuando las empresas exportan.

\section{Probabilidad de exportar según el nivel de productividad}

Para medir la probabilidad de exportar según el nivel de productividad de las pymes, se estiman cómo las diferentes características económicas y de las exportaciones realizadas en el pasado afectaron probabilísticamente esas decisiones de las pymes. De igual manera se examina, cómo las probabilidades medias de exportar se relacionaron con los diferentes rangos de las productividades laborales y cómo éstas evolucionaron durante los doce años objeto de estudio. 
Econométricamente se estiman cuatro modelos probabilísticos probit, ${ }^{1}$ siendo la variable dependiente la dummy de si exporta con valor de uno y cero si no lo hizo. Las variables explicativas de los modelos se relacionan con el logaritmo de los indicadores económicos usados en los modelos anteriores, como son: la productividad laboral, el capital y el total de ocupados. También se evalúa el efecto de la proporción de los trabajadores en producción en términos del total, 22 dummies de actividad económica y el rezago de la variable dependiente dicotómica de si exportó en el período anterior (Exportó_1). Además, se examina el efecto de la variable

$$
\left(1-\text { Exportó }_{-1}\right) \bullet \text { Exportó }_{-2},
$$

que indica si una pyme exportó en los dos períodos anteriores, pero no en el inmediatamente anterior (Bernard \& Jensen, 2004).

Se eligieron tres rangos de probabilidad (alta, media y baja), con el criterio de que el número de empresas no estuviera concentrado en un subgrupo pequeño de pymes y además se corrigieron los errores por el método robust cluster de agrupamiento robusto, para permitir mayor eficiencia en la convergencia de la maximización de la función de verosimilitud.

Los parámetros estimados con los indicadores económicos de las empresas muestran que existen relaciones probabilísticas positivas y estadísticamente significativas al $1 \%$. El signo con la proporción de los trabajadores no calificados en relación con el total es negativo, como es lo esperado. Las modelos probit (1) y (2) se estiman con la productividad laboral medida por el valor agregado y los modelos (3) y (4) con la productividad laboral en términos de la producción manufacturada. El tamaño de las pymes se mide alternativamente con el logaritmo del capital o con el del total de los ocupados.

Los efectos marginales de las estimaciones de los modelos probit (Tabla 9) indican que un aumento en el $1 \%$ del logaritmo de las productividades laborales aumenta la probabilidad de exportar de una pyme entre 1 y $2 \%$, mientras un crecimiento del $1 \%$ en su tamaño, medido por el logaritmo de los

1 Son estimaciones no lineales, donde la variable dependiente es dicotómica. La función probit transforma la variable dicotómica $Y$ en una distribución normal acumulativa. Entonces la estimación es una distribción normal acumulativa, con rango entre 0 y 1. 
Mesa y Torres: Pymes manufactureras exportadoras en Colombia: análisis de su actividad real

ocupados, aumenta en cerca de $2 \%$ la probabilidad de exportar. En cambio, si el tamaño se mide por el logaritmo del capital, su aumento en $1 \%$ incrementa las probabilidades en menos de $1 \%$.

Tabla 9. Efectos probit marginales de exportar por las pymes: 2000-2001.

Variable dependiente es la Dummy con uno para las exportadoras

\begin{tabular}{lcccc}
\hline Variables & $(1)$ & $(2)$ & $(3)$ & $(4)$ \\
exporta & exporta & exporta & exporta \\
\hline $\begin{array}{l}\text { Log. productivida valor } \\
\text { agregado por trabajador }\end{array}$ & $\begin{array}{c}0132^{* * *} \\
(0,001)\end{array}$ & $\begin{array}{c}\left(0,0121^{* * *}\right. \\
(0,0012)\end{array}$ & & \\
Log. productivida producción & & & $0,0146^{* * *}$ & $0,012^{* * *}$ \\
industrial por trabajador & & & $(0,001)$ & $(0,0013)$ \\
No calificados/total ocupados & $-0,0114^{* * *}$ & & $-0,0099^{* *}$ & \\
& $(0,0038)$ & & $(0,0039)$ & \\
Log. total ocupados & $0,0222^{* * *}$ & & $0,0234^{* * *}$ & \\
& $(0,0013)$ & & $(0,0013)$ & \\
Log. del capital & & $0,006^{* * *}$ & & $0,0051^{* * *}$ \\
& & $(0,0009)$ & & $(0,001)$ \\
Dummy uno si exportó el & $0,244^{* * *}$ & $0,2623^{* * *}$ & $0,2418^{* * *}$ & $0,2646^{* * *}$ \\
período anterior & $(0,0044)$ & $(0,0045)$ & $(0,0044)$ & $(0,0045)$ \\
Dummy uno si exportó dos & $0,180^{* * *}$ & $0,194^{* * *}$ & $0,1793^{* * *}$ & $0,1962^{* * *}$ \\
períodos antes pero no en el & $(0,0034)$ & $(0,0034)$ & $(0,0033)$ & $(0,0034)$ \\
período anterior & & & & 72,338 \\
\hline Observaciones & 73,895 & 72,335 & 73,894 & 11,946 \\
Número de empresas & 11,965 & 11,946 & 11,963 & \\
\hline
\end{tabular}

Nota: errores estándar entre paréntesis. ${ }^{* * *} \mathrm{p}<0,01,{ }^{* *} \mathrm{p}<0,05,{ }^{*} \mathrm{p}<0,1$.

Fuente: estimaciones con base en la EAM del DANE.

Si las pymes exportaron el año previo, la probabilidad de que lo sigan haciendo con respecto a las que no lo hicieron es significativamente alta (alrededor del $24 \%$ ), y si lo hicieron dos años antes, pero no en el período inmediatamente anterior, la probabilidad es menor pero también alta (cercana 
al 18 \%). Como lo señalan Clerides, Lach y Tybout (1998), el efecto de la experiencia previa de exportar es sustancial en todas las actividades industriales, y más para las empresas que exportaron en el año previo.

El análisis probabilístico de exportar de una pyme para el período 20002012 según la productividad laboral, medida por el valor agregado por trabajador, se aproxima en este ensayo a través del análisis gráfico. Puesto que los gráficos de exportar según los rangos de los logaritmos de las productividades laborales son similares en los cuatro modelos probit que se estimaron, sólo se presenta el gráfico del modelo probit (1) (Gráfico 6).

Gráfico 6. Probabilidad de las pymes de exportar, 2000-2012

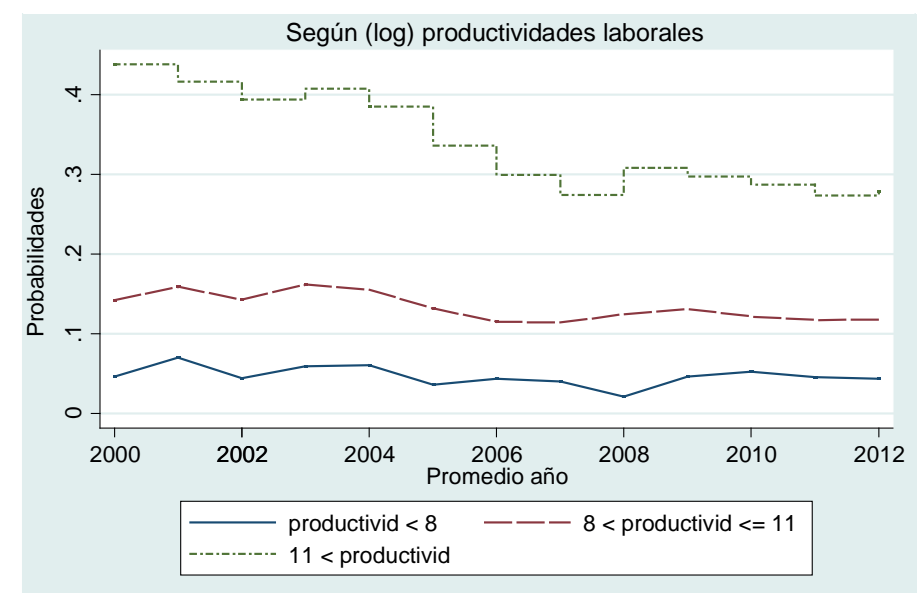

Fuente: estimaciones con base en la EAM del DANE.

Para rangos de baja productividad laboral, menores al logaritmo de 8 , la probabilidad de exportar es cercana a $7 \%$ y con leve tendencia decreciente. En niveles medios de productividad laboral, entre logaritmos 8 y 11, las probabilidades se duplican y mantienen su nivel. Para niveles altos de productividad laboral, logaritmos mayores de 11 , las probabilidades son cercanas al $40 \%$, pero éstas cayeron y se acercaron a $30 \%$ el final del período. 
Mesa y Torres: Pymes manufactureras exportadoras en Colombia: análisis de su actividad real

\section{Conclusiones}

La evidencia empírica que se organizó para estudiar la propensión a exportar de las empresas pequeñas y medianas en Colombia permite confirmar la modesta orientación de la estructura industrial colombiana hacia los mercados internacionales. El porcentaje de empresas que se involucra en el comercio internacional es relativamente bajo y la dinámica de salida es alta. Es importante indicar que en este período la expansión de la economía colombiana hizo rentable el mercado local a expensas del externo, donde predominó la revaluación de la moneda nacional frente al dólar.

Las actividades industriales que mostraron la mayor participación de las pymes exportadoras fueron: cuero y calzado, metalúrgica básica (con exportaciones sospechosas), aparatos de radio y televisión y equipo de transporte. Sin embargo las actividades industriales con menor participación en número de empresas, pero con mayor participación de sus valores exportados, fueron las industrias de alimentos, bebidas y tabaco.

Las pymes que exportaron crecieron más en términos de su producción industrial que las no exportadoras, además fueron las que presentaron los mayores tamaños en términos de capital y número total de ocupados. De manera evidente las pymes exportadoras presentaron menores relaciones de trabajo no calificado a calificado con respecto al conjunto de estas empresas.

Existe alta probabilidad de que las empresas que exportaron continúen haciéndolo y expliquen en mayor medida el crecimiento de las exportaciones. La variable que explica la orientación exportadora de las pymes es el nivel de productividad, en este caso la laboral. Las empresas que registraron los mayores niveles de productividad presentaron altas probabilidades de exportar, cerca de $40 \%$, aunque esa probabilidad registró una tendencia decreciente en el período 2000-2012, quizás debido a la revaluación de la moneda nacional frente al dólar y a los problemas políticos y económicos que se dieron con los países fronterizos, donde se realizaban en parte esas exportaciones.

Falta abordar el tema del destino de las exportaciones de las pymes. Diferentes estudios señalan que el conjunto de empresas exportadoras colombianas lo hacen a un mercado, normalmente a los países vecinos o a los Estados 
Unidos. Además las empresas colombianas explotan intensamente sus productos principales con escasa sofisticación (sus márgenes intensivos), que las conduce a rivalizar intensamente en los mercados externos muy competidos. Sólo se escalarían las exportaciones de estas empresas con la mayor diferenciación de sus variedades y mejores calidades de sus mercancías, tema que corresponde a otra investigación.

Es indiscutible que el crecimiento y la diversificación de las exportaciones de la economía colombiana, con sus efectos en términos de empleo y desarrollo económico, se vincularán con el desempeño exportador de las pymes en las diversas regiones colombianas. La importancia de las empresas exportadoras radica en que su inserción en los mercados externos las podría estimular en el mediano y largo plazo a introducir nuevas tecnologías en sus procesos productivos, mejorar la calidad de sus productos y aprovechar las economías de escala. Lo anterior llevaría como resultado al aumento de la productividad del conjunto de la economía. Esta última hipótesis no se ha validado empíricamente.

\section{Referencias}

Bernard, Andrew B. \& Jensen, J. Bradford (1999). "Exceptional exporter performance: cause, effect, or both?”, Journal of International Economics, Vol. 47, pp. 1-25.

Bernard, Andrew B. \& Jensen, J. Bradford (2004). "Why Some Firms Export", The Review of Economics and Statistics, Vol. 86, No. 2, 561-569.

Bernard, Andrew B.; Jensen, J. Bradford \& Kortum, Samuel S. (2003). "Plants and productivity in international trade", American Economic Review, Vol. 93, No. 4, pp. 1268-1290.

Bernard, Andrew B.; Jensen, J. Bradford; Redding, Stephen J. \& Schott, Peter K. (2007). "Firms in International Trade", The Journal of Economic Perspectives, Vol. 21, No. 3, pp. 105-130.

Brooks, Eileen L. (2006). "Why don't firms export more? Product quality and Colombian plants", Journal of Development Economics, Vol. 80, No. 1, pp. 160-178. 
Mesa y Torres: Pymes manufactureras exportadoras en Colombia: análisis de su actividad real

Clerides, Sofronis; Lach, Saul \& Tybout, James (1998). "Is Learning by Exporting Important? Micro-Dynamic Evidence from Colombia, Mexico, and Morocco", The Quarterly Journal of Economics, Vol. 113, No. 3, pp. 903-947.

Correa, Alexander \& RoA, Santiago (2012). “Diferencias en el desempeño exportador de las Pymes colombianas", Revista Civilizar de Empresa y Economía, Vol. 3, No. 5, 62-80.

Eaton, Jonathan; Eslava, Marcela; Hernández, Mónica; Kugler, Maurice; Montes, Enrique; Rueda, Miguel \& Tyвout, James (2007). "Mercados de exportación: entramos, salimos, nos quedamos", Revista del Banco de la República, Vol. 80, No. 951, pp. 10-27.

Eaton Jonathan; Kortum, Samuel \& Kramarz, Francis (2004). "Dissecting Trade: Firms, Industries, and Export Destinations", The American Economic Review, Vol. 94, No. 2, pp. 150-154.

Helpman, Elhanan; Melitz, Marc \& Yeaple, Stephen (2004). "Export versus FDI with Heterogeneous Firms", The American Economic Review, Vol. 94, No. 1, pp. 300-16.

LAWless, Martina (2009). "Firm export dynamics and the geography of trade", Journal of International Economics, Vol. 77, No. 2, pp. 245-254.

Mesa, Fernando \& Perilla, Juan Ricardo (2007). "Exportaciones óptimas y políticas comerciales para las industrias de textiles y de confecciones: casos de Colombia y México 1989-2002", El Trimestre Económico, Vol. 74, No. 293, pp. 195-221.

Meléndez, Marcela (2013). "Internacionalización de las Pymes en Colombia”, Revista Integración \& Comercio, Vol. 37, No. 17, pp. 25-40.

Melitz, Marc J. (2003). “The Impact of Trade on Intra-Industry Reallocations and Aggregate Industry Productivity", Econometrica, Vol. 71, No. 6, pp. 1695-1725.

Melitz, Marc J. (2008) "International Trade and Heterogeneous Firms. En: Durlauf, S. \& Blume, L. (Eds.), New Palgrave Dictionary of Economics. New York: Macmillan Publisher. 
Milesi, Darío; Moori, Virginia; Verónica, Robert \& Yoguel, Gabriel (2007). "Desarrollo de ventajas competitivas: pymes exportadoras exitosas en Argentina, Chile y Colombia”, Revista de la CEPAL, Vol. 92, 25-43.

Milesi, Darío \& Aggio, Carlos (2008). "Éxito exportador, innovación e impacto social: un estudio exploratorio de Pymes exportadoras latinoamericanas", Working Paper, FUNDES-BID.

Moori, Virginia; Rodríguez, Astrid; Yoguel, Gabriel \& Granados, Amanda (2005). Perfil de las Pymes exportadoras exitosas: El caso colombiano. Bogotá: FUNDES.

Nassimbeni, Guido (2001). "Technology, innovation capacity, and the export attitude of small manufacturing firms: a logit/tobit model”, Research Policy, Vol. 30, No. 2, pp. 245-262.

Roberts, Mark \& Tyвоut, James (1997). “The Decision to Export in Colombia: An Empirical Model of Entry with Sunk Costs", The American Economic Review, Vol. 87, No. 4, pp. 545-64.

Uribe, Claudia M.; Ardila, Gustavo \& Esmerald, Fernando (2006). Financiamiento y promoción a la PYME Exportadora: Caso colombiano. Perú: Programa ALIDE-BID-FOMIN. 